
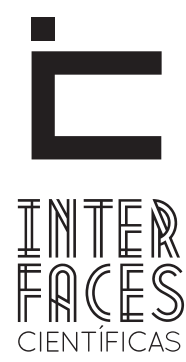

SAÚDE E AMBIENTE

\title{
ANÁLISE QUALITATIVA DA SÍNDROME DE BURNOUT NOS ENFERMEIROS DE SETORES ONCOLÓGICOS
}

Mayara Torquato da Silva ${ }^{1}$

\section{RESUMO}

A Síndrome de Burnout é uma forma de resposta ao estresse laboral crônico, sendo esta uma condição na qual o trabalhador se desgasta, e desiste, na medida em que perde a satisfação e sentido pelo trabalho. 0 presente estudo teve como objetivo analisar alguns artigos científicos que abordam a presença da Síndrome de Burnout nos enfermeiros que trabalham no setor de oncologia. Trata-se de uma pesquisa de revisão de literatura integrativa realizada com artigos indexados em sites de cunho científico por meio dos descritores: Síndrome de Burnout, enfermeiros, oncologia. Por meio deste estudo constatou-se que o ambiente hospitalar e o setor de oncologia são locais
Fernanda Gomes de Magalhães²

geradores de grandes cargas de estresse, e fatores predisponentes aos profissionais, principalmente os enfermeiros que estão em contato maior com 0 paciente a desenvolverem a Síndrome de Burnout. Conclui-se que existe a necessidade de maiores pesquisas sobre a síndrome, principalmente no setor oncológico, ressalta as condições de trabalho destes profissionais e um foco à saúde física e mental dos trabalhadores.

\section{PALAVRAS CHAVES}

Síndrome de Burnout. Enfermeiros. Oncologia. 


\section{ABSTRACT}

The burnout syndrome is a form of response to chronic job stress, which is a condition in which the worker wears and gives up, in that it loses meaning and satisfaction at work. This article aims to analyze scientific articles that addresses the presence of burnout in nurses working in an oncology ward. This is a survey conducted by indexed articles on sites through scientific nature of descriptors: Burnout Syndrome, nurses, oncology. Through this study it was found that the hospital and the oncology sector are generating heavy burden and factors that predisposing the professionals, especially nurses who are in greater contact with the patient to develop a burnout syndrome. We conclude that there is a need for further research on the syndrome, especially in oncology sector, highlighting the working conditions of these professionals and a focusing the physical and mental health of the workers.

\section{KEYWORDS}

Burnout. Nurses. Oncology.

\section{RESUMEN}

El síndrome de desgaste es una forma de respuesta a la tensión de trabajo crónica, que es una condición en la que el trabajador lleva y abandona, en que pierde significado y la satisfacción en el trabajo. El presente estudio tuvo como objetivo analizar los artículos científicos que se ocupan de la presencia del síndrome de burnout en enfermeras que trabajan en una sala de oncología. Esta es una encuesta de la revisión bibliográfica realizada con artículos integradoras sitios a través de la naturaleza científica de los descriptores indexados: Síndrome de Burnout, enfermeras, oncología. A través de éste estudio se encontró que el hospital y los sitios del sector de oncología están generando grandes cargas de estrés y los factores que predisponen a los profesionales, especialmente de enfermeras que están en mayor contacto con el paciente para desarrollar un síndrome de burnout. Llegamos a la conclusión de que hay una necesidad de más investigación sobre el síndrome, sobre todo en el sector de la oncología, dice que las condiciones de trabajo de estos profesionales y un enfoque en los trabajadores de la salud física y mental.

\section{PALABRAS CLAVES}

Burnout. Enfermeras. Oncología.

\section{INTRODUCÇ̃O}

A enfermagem é considerada a arte do cuidar, uma vez que os enfermeiros são profissionais que permanecem maior tempo junto ao paciente, dentro dos diversos contextos do cuidar, prestando-lhe o cuidado integral. Contudo, o ambiente hospitalar, talvez traga consigo impactos à saúde do trabalhador, manifestando-se tanto na esfera física, como na psíquica. Além disso, o contato próximo com o paciente pode gerar conflitos emocionais, possibilitando ao profissional de enfermagem um sofrimento psíquico (et al., 2012).
O exercício profissional no âmbito hospitalar é marcado por múltiplas exigências: lidar com dor, sofrimento, morte e perdas, a que se somam as condições desfavoráveis de trabalho e a baixa remuneração, fatores que, em conjunto, propiciam a emergência de estresse e burnout, termo criado para descrever o desgaste físico e psíquico de profissionais que lidam no exercício de suas funções, com altos níveis de envolvimento emocional. 0 setor de oncologia porta uma particularidade, que 
são as representações do câncer, fortemente associadas à ideia de morte (AVELLAR; IGLESIAS; VALVERDE, 2007).

Estar/assistir a um indivíduo com câncer, embora recompensador, tanto pelos benefícios que podemos propiciar ao paciente como pelos que ele pode oferecer ao profissional, como indivíduos e cuidadores, faz refletir sobre valores, projetos de vida, medos, sonhos, atitudes e consequências do que já se fez ou poderia ter sido feito, enfim, abre a porta para um turbilhão de emoções que nem sempre deseja-se viver em determinados momentos da vida (CAMPOS, 2005).

A Síndrome de Burnout é uma forma de resposta ao estresse laboral crônico, sendo esta uma condição na qual o trabalhador se desgasta, e desiste, na medida em que perde a satisfação e sentido pelo trabatho (MATUBARO et al., 2010). Já de acordo com Codo e Menezes (2002) é definida como uma condição na qual o trabalhador perde o sentido da sua relação com o trabalho, de forma que as coisas já não importam mais e qualquer esforço lhe parece ser inútil. Também apontam que a síndrome é entendida como um conceito multidimensional que envolve três componentes: (1) exaustão emocional, (2) despersonalização e (3) falta de envolvimento pessoal no trabalho.

Esta síndrome manifesta-se por meio de quatro classes sintomatológicas, sendo: física, quando o trabalhador apresenta fadiga constante, distúrbio do sono, falta de apetite e dores musculares; psíquica observada pela falta de atenção, alterações da memória, ansiedade e frustração; comportamental, identificada quando o indivíduo apresenta-se negligente no trabalho, com irritabilidade ocasional ou instantânea, incapacidade para concentrar-se, aumento das relações conflitivas com os colegas, longas pausas para o descanso, cumprimento irregular do horário de trabalho; e defensiva, quando o trabalhador tem tendência ao isolamento, sentimento de onipotência, empobrecimento da qualidade do trabalho e atitude cínica (JODAS; HADDAD, 2008).

A motivação desta pesquisa ocorreu devido ao grande desgaste físico e emocional dos enfermeiros que trabalham no setor oncológico, presenciado no decorrer dos estágios da graduação e pós-graduação da enfermagem.

Por ser pouco conhecida pelos profissionais de saúde, há necessidade de maiores estudos sobre a Síndrome de Burnout, visto que quando os trabalhadores desconhecem as manifestações e causas desse fenômeno, não buscam formas efetivas de prevenção ou intervenção. Desta Maneira, o presente estudo teve como objetivo analisar por meio da revisão de literatura a presença da Síndrome de Burnout em profissionais de saúde no setor de oncologia.

Para o estudo de revisão integrativa buscaram-se artigos, livros e revistas científicas acerca da Síndrome de Burnout em enfermeiros do setor oncológico, operacionalizado mediante busca eletrônica de artigos indexados nas seguintes bibliotecas virtuais: SCIELO - Scientific Electronic Library Online (http:// www.scielo.org) e LILACS - Literatura Latino - Americana e do Caribe em Ciências da Saúde (http://li- 
lacs.bvsalud.org/), BVS- Biblioteca Virtual em Saúde (http://bases.biereme.br).

Primeiramente as obras foram pesquisadas, armazenadas e em seguida, realizada a pré-seleção com critério de inclusão artigos que continham os descritores: Síndrome de Burnout (SB), Enfermeiros e Oncologia, e com período de publicação entre o ano de 1996 a 2013, disponíveis na base de dados em língua portuguesa. Foram excluídos os trabalhos que não apresentavam relação com o objeto de estudo, que não estavam disponíveis on-line, e que não estiveram dentro do período de tempo delimitado.

$\mathrm{Na}$ coleta de dados foram localizados 22 artigos para discussão. 0 material selecionado foi organizado na tabela a seguir (tabela 1).

TABELA 1 - Relação quantitativa de obras sobre a temática, levantadas em ambiente virtual em 2013

\begin{tabular}{|c|c|c|c|c|c|}
\hline TIPO MATERIAL & $\begin{array}{c}\text { FONTE } \\
\text { PESQUISADA }\end{array}$ & ANO & $\begin{array}{l}\text { QUANTIDADE } \\
\text { DE ARTIGOS }\end{array}$ & $\begin{array}{c}\text { TIPO } \\
\text { ESTUDO }\end{array}$ & TOTAL \\
\hline Artigos & $\begin{array}{l}\text { Scielo :10 } \\
\text { Site Unifra: } 01 \\
\text { Site Ufpa: } 01 \\
\text { Site Aprendizagem S } \\
\text { ignificativa: } 01 \\
\text { Revista eficaz: } 01 \\
\text { BVS: } 01 \\
\text { Revista Proteção: } 01\end{array}$ & $\begin{array}{l}\text { 2002: } \\
\text { 2005: } \\
\text { 2007: } \\
\text { 2008: } \\
\text { 2009: } \\
\text { 2010: } \\
\text { 2011: } \\
\text { 2012: }\end{array}$ & $\begin{array}{l}02 \\
02 \\
02 \\
01 \\
03 \\
02 \\
01 \\
02\end{array}$ & $\begin{array}{l}\text { RB: } 06 \\
\text { PC: } 08 \\
\text { MTO: } 02\end{array}$ & 16 \\
\hline Manuais e Outros & $\begin{array}{l}\text { Site MS: } 01 \\
\text { Site UCG: } 01\end{array}$ & $\begin{array}{l}2001 \\
2010\end{array}$ & $\begin{array}{l}01 \\
01\end{array}$ & $\begin{array}{l}\text { PC: } 01 \\
\text { RB: } 01\end{array}$ & 02 \\
\hline Teses & $\begin{array}{l}\text { BVS: } 01 \\
\text { Site Repositório Aberto: } 01 \\
\text { Site UNIRIO: } 01\end{array}$ & $\begin{array}{l}\text { 2005: } \\
2010 \\
2007\end{array}$ & $\begin{array}{l}01 \\
01 \\
01\end{array}$ & $\begin{array}{l}\text { PC: } 02 \\
\text { MTO: } 01\end{array}$ & 03 \\
\hline
\end{tabular}

RB: Revisão Bibliográfica

PC: Pesquisa de Campo

MTO: Misto (possuem os dois tipos de estudo)

MS: Ministério da Saúde

UCG: Universidade Católica de Goiás

UNIFRA: Centro Universitário Franciscano

UFPA: Universidade Federal do Pará

UNIRIO: Universidade Federal do Estado do Rio de Janeiro

Após a análise da literatura foram selecionadas, na base de dados e leitura sistemática, duas categorias. A primeira relacionada a aspectos histórico e social da
Síndrome de Burnout e a segunda referente ao estresse, esgotamento profissional e ambiente de trabalho na Enfermagem Oncológica, que serão explicadas a seguir. 


\section{RESULTADOS E DISCUSSÕES}

\subsection{ASPECTOS HISTÓRICO E SOCIAL DA SÍNDROME DE BURNOUT}

O termo burnout consiste em uma conjunção entre "burn" e "out”, ambas as palavras da língua inglesa, onde a primeira significa "arder", "queimar", enquanto a segunda se refere a "fora", "para fora" (MICHAELIS, 1989). Portanto, o significado literal para burnout em português é “queimar para fora”. Porém, sua tradução é mais bem expressada pela palavra esgotamento.

O primeiro autor a descrever e conceituar a Síndrome de Burnout foi Freudenberger, em 1974. Contudo, foram Maslach \& Jackson que, ainda no final da década de 1970 e início dos anos 1980, elaboraram tanto um modelo teórico quanto um inventário para a mensuração da síndrome (SOUZA; SILVA, 2002).

Inicialmente foi diagnosticado em indivíduos que pertencem às chamadas profissões de ajuda (helping professions). Advogados, professores, assistentes sociais, dentistas, enfermeiros e médicos são exemplos desse tipo de profissões, que se caracterizam por constantes relações interpessoais, bem como pela necessidade do profissional em promover avanços de ordem biológica, psicológica, social, intelectual e/ ou financeira em seu cliente/paciente/aluno (PIRES; BRANDÃO; MACHADO, 2005).

0 estudo da literatura internacional indica que não existe uma definição única sobre Burnout, mas é consenso até os estudos hoje desenvolvidos que seria uma resposta ao stress laboral crônico, não devendo, contudo ser confundido com stress. 0 primeiro envolve atitudes e condutas negativas com relação aos usuários, clientes, organização e trabalho; é assim, uma experiência subjetiva, envolvendo atitudes e sentimentos que vêm acarretar problemas de ordem prática e emocional ao trabalhador e à organização. 0 conceito de stress, por outro lado, não envolve tais atitudes e condutas, é um esgotamento pessoal com interferência na vida do indivíduo e não necessariamente na sua relação com o trabalho (CODO; MENEZES, 2002).

A Síndrome de Burnout (SB) constitui-se de três dimensões: Exaustão Emocional, Despersonalização e Baixa Realização Profissional. A Exaustão Emocional se caracteriza por uma falta ou carência de energia, entusiasmo e um sentimento de esgotamento de recursos. A Despersonalização caracteriza-se por tratar os clientes, colegas e a organização de forma distante e impessoal. A Baixa Realização Profissional é a tendência do trabalhador em se autoavaliar de forma negativa. As pessoas se sentem infelizes e insatisfeitas com seu desenvolvimento profissional e experimentam um declínio no sentimento de competência e êxito no trabalho (CARLOTTO, 2011 apud MASLACH; SCHAUFELI; LEITER, 2001).

A SB vem sendo considerada problema de saúde pública, devido a suas implicações para a saúde física e mental do trabalhador, com evidente comprometimento de sua qualidade de vida no ambiente de trabalho, e ainda constitui um dos grandes problemas psicossociais da atualidade, e reflete o modo de vida capitalista, baseado na lógica dos meios de produção e do consumo desenfreado (SILVA, DIAS, TEIXEIRA, 2012).

Ainda que o termo Burnout não esteja tão disseminado e popularizado quanto o Estresse, ele precisa ser considerado como um problema internacional, não sendo um privilégio de uma específica realidade social, educacional ou cultural. É uma síndrome que vem acometendo os trabalhadores desde o final do século passado e continua neste novo milênio. É preciso compreender que as transformações no mundo do trabalho implicaram, também, mudanças nas relações sociais e de trabatho, afetando o bem-estar físico e mental dos trabathadores e dos grupos sociais dos quais eles fazem parte (MUROFUSE; ABRANCHES; NAPOLEÃO, 2005). 
CAMPOS (2005) Define que a Síndrome está inserida na Lista de Doenças Relacionadas ao Trabalho com a denominação de Sensação de Estar Acabado - Síndrome de Burnout ou Síndrome do Esgotamento Profissional - CID- 10, Z73.0. A lista é organizada segundo grupos de patologias ou processos mórbidos da Classificação Internacional das Doenças e consta da décima revisão, aprovada pela Portaria/MS no 1.339/1999. As doenças são organizadas em um sistema de dupla entrada, por doença ou por agente etiológico ou por fator de risco de natureza ocupacional. A Síndrome de Burnout está inserida no Grupo V, nos Transtornos Mentais e do Comportamento Relacionado ao Trabalho.

Esta doença já é reconhecida como doença relacionada ao trabalho, sendo contemplada na Lista B do Regulamento da Previdência, estabelecido pelo Ministério da Previdência e Assistência Social do Brasil (Ministério da Saúde, 2001).

Em uma pesquisa realizada pela International Stress Management Association do Brasil (ISMA-BR) com mil profissionais de São Paulo e Porto Alegre, com idades entre 25 a 60 anos, revela que no Brasil o problema atinge $30 \%$ da População Economicamente Ativa (PEA). Os dados mostram ainda que, dos $30 \%$ dos entrevistados que sofrem de Burnout, $94 \%$ se sentem incapacitados para trabalhar; $89 \%$ praticam presenteísmo - o que significa que estão presentes no trabalho, mas não conseguem realizar as tarefas propostas - e $47 \%$ sofrem de depressão. 0 estudo fez ainda um comparativo do desempenho profissional de um portador de Burnout com o dos demais trabalhadores: em média, é de menos cinco horas. Entre os sintomas de Burnout que mais incomodam, 93\% alegam a exaustão; $86 \%$, a irritabilidade; $82 \%$ a falta de concentração; e $74 \%$ enfatizam a dificuldade de relacionamento no ambiente profissional (O GLOBO ONLINE, 2010).

\subsection{ESTRESSE, ESGOTAMENTO PROFISSIONAL E AMBIENTE DE TRABALHO NA ENFERMAGEM ONCOLÓGICA}

A enfermagem é responsável pelo maior contingente da força de trabalho dos estabelecimentos hospitalares, com responsabilidade pela assistência nas 24 horas, configurando-se como o conjunto de trabalhadores que mais sofre com as inadequadas condições de trabalho e com a insalubridade do ambiente (COSTA; VIEIRA; SENA, 2009).

0 ambiente hospitalar funciona como gerador de estresse tanto aos pacientes e seus familiares, devido à enfermidade e à situação de internação, quanto aos profissionais que ali atuam, com isso o profissional é suscetível ao fenômeno do estresse ocupacional (SILVA; DIAS; TEIXEIRA, 2012).

Assim este ambiente é o local em que o enfermeiro fica exposto a riscos biológicos e químicos, sofre forte carga emocional e física, atua em horários atípicos, com longas jornadas de trabalho, insuficiência de funcionários, carência de materiais e equipamentos, baixos salários, sem autonomia e motivação (CINTRA et al., 2009). Com isso Nogueira (2007) em sua pesquisa sobre causas de afastamentos do trabalho por adoecimento do profissional de enfermagem do ambulatório de uma instituição oncológica em um hospital do Rio de Janeiro, afirma que o ambiente de trabalho do enfermeiro oncologista é o hospital, com isso é importante notar que a dinâmica organizacional do trabalho em hospitais gera grande tensão ocupacional, sendo necessário monitorar periodicamente a saúde mental e física desses trabalhadores (MATUBARO et al., 2010).

No entanto, não podemos deixar de apontar as dificuldades dos profissionais da área hospitalar na lida diária com o sofrimento humano. Acredita-se que estas dificuldades se superlatizam quando transportadas para os serviços de atendimento a pacientes críticos ou com doença crônico-degenerativa que os 
obrigam a longos períodos de internação ou a vários retornos ao hospital como é o caso dos pacientes oncológicos (FERREIRA, 1996).

O setor oncológico, de fato, é um local carregado de fatores desencadeantes de estresse e outras alterações emocionais (VIANA, 2010). A rotina neste setor é descrita como acelerada, com múltiplas demandas, resultantes de um ambiente de trabalho tido como desgastante e muito estressante. Após algum tempo o trabalhador já não se reconhece como era anteriormente ao ingresso no setor oncológico, como se surgisse um sentimento de despersonalização, resultante de sua insatisfação com a função que exerce e com o lugar em que está inserido (AVELAR; IGLESIAS; VALVERDE, 2007).

A Enfermagem foi classificada pela Health Education Authority como a quarta profissão mais estressante. Além disso, encontra dificuldades em delimitar os diferentes papéis da profissão e, consequentemente, a falta de reconhecimento nítido entre o público, elevando a despersonalização do trabalhador em relação à profissão (JODAS; HADDAD, 2009)

A profissão de enfermagem e seus trabalhadores são caracterizados por terem, em sua essência, o cuidado; tendo um contato direto com pacientes e familiares, ou seja, lidam, constantemente, com pessoas com as mais diversas concepções e cultura. Esse tipo de relação contribui fortemente para a gênese do estresse laboral. A síndrome surge nos profissionais de enfermagem de todo o mundo, em diferentes contextos de trabalho, levando-os a desenvolverem sentimentos de frustração, frieza e indiferença em relação às necessidades e ao sofrimento dos doentes (SILVA; DIAS; TEIXEIRA, 2012).

Para Carlotto (2011, apud Gil-Monte 2002) relata que apesar de um aumento no número de profissionais homens nas equipes de enfermagem, a profissão, ainda, é tradicionalmente vinculada ao gênero feminino em virtude das suas características de cuidado. Entretanto na pesquisa feita pelo autor mencionado acima, com técnicos de enfermagem em três hospitais de Porto Alegre. Observou-se que com relação às variáveis sociodemográficas, que mulheres apresentaram maior exaustão emocional, despersonalização e menor realização profissional, sintomas que são observados na Síndrome de Burnout. Contudo na revisão bibliográfica feita por Viana (2010) constatou-se que a maioria dos profissionais atuantes no setor oncológico é formada por mulheres e que não escolheram atuar nesse setor, elevando assim fatores contribuintes para o sofrimento emocional, e consequentemente apresenta fator de risco para o aparecimento desta Síndrome.

Os profissionais de saúde, em particular, os enfermeiros estão predispostos a desenvolver Burnout pelas características do seu trabalho: trabalham diretamente e intensamente com pessoas em sofrimento. Dentre os profissionais de saúde são os que mais tempo passam com os pacientes nas unidades de internamento ou de tratamento em ambulatório. Em particular, os enfermeiros que trabalham nos setores da oncologia, muitas das vezes, se sentem esgotados pelo fato de darem muito de si aos seus doentes e em compensação, pelas características da doença, receberem muito pouco (CUMBE, 2010).

O exercício da enfermagem em oncologia requer atividades de controle e exercício mental maiores que em outras áreas, uma vez que implica lidar com doença grave, cuidar de pacientes fora de possibilidades terapêuticas e terminais, a necessidade de cuidados intensivos e, também, prolongados e a elevada proximidade com a família, com vivência de situações emocionais desgastantes para o profissional. Esses fatores considerados estressantes pelos enfermeiros, que atuam em oncologia devem ser considerados potenciais desencadeadores da síndrome de Burnout (RODRIGUES; CHAVES, 2008).

Para os profissionais da enfermagem atuante no setor oncológico, o cuidar transpõe a terapêutica, causando assim um esforço subumano por parte do 
cuidador, que lida com seus limites emocionais em seu cotidiano. Inúmeros são os fatores que levam a equipe de enfermagem a desenvolver o estresse no setor em que atua, como o desgaste emocional devido o sofrimento psíquico adquirido com fatores de onipotência frente à morte, o sofrimento dos familiares e pacientes e o estigma do câncer, são fatores que levam o cuidador a desenvolver não somente o estresse ocupacional, mas também várias outras patologias que colocam em risco a saúde física e mental desse cuidador (VIANA, 2010).

Em sua pesquisa bibliográfica o mesmo autor afirma que são poucos profissionais que escolhem atuar em setores críticos, a oncologia é considerada crítica por ser um setor que lida diariamente com o sofrimento de pacientes sem probabilidade terapêutica, e com sobrecarga emocional, mas este fato deve ser levado em consideração quando o cuidador é designado para atuar neste setor, caso contrário será um estressor com grande potencial na vida do individuo.

Uma pesquisa feita por Avellar; Iglesias; Valverde (2007) com profissionais de enfermagem que tra- balham em uma unidade oncológica retrataram os sentimentos relatados por estes, onde vivenciaram momentos de tristeza, vivências de mal-estar, sentimento de impotência e depressão.

No entanto, mais do que relatar sentimentos, o que ocorreu com frequência foi a tentativa de fazer com os sentimentos citados acima não aparecessem na vida profissional destes trabalhadores. O envolvimento com o paciente é percebido como algo desgastante, especialmente nos casos em que a possibilidade de cura é remota; traduz-se em sentimentos de impotência, frustração, tristeza e chateação. A estratégia de não se apegar ao paciente leva a um conflito, em decorrência de, muitas vezes, não se obter êxito nesta tarefa.

Em um estudo realizado por Ramalho; Martins (2007) com diversos profissionais (dois médicos, um secretário, um psicólogo, um farmacêutico, um enfermeiro e três auxiliares de enfermagem) que atuam numa clínica de oncologia pediátrica, apontou como fontes de estresse as dificuldades da organização do trabalho, bem como as características da doença, seu tratamento e a morte de crianças.

\section{CONCLUSÃO}

Diante do estudo bibliográfico realizado, observou-se que a Síndrome de Burnout é pouco conhecida pelos profissionais de saúde, e existem na literatura poucos artigos sobre esta patologia, principalmente quando foca na profissão de enfermagem, e na área de oncologia, e ainda verificaram estudos recentes sobre este tema, sendo a maioria com publicações dos últimos 15 anos.

Sugere que sejam realizadas pesquisas posteriores com foco na área de oncologia, já que Viana (2010) afirma que o setor oncológico, de fato, é um local carregado de fatores desencadeantes de

estresse e outras alterações emocionais, considerando assim um ambiente favorável para o aparecimento de Burnout.

Outra variável encontrada nos estudos foi que os indivíduos de profissões de ajuda, como é o caso da enfermagem que estão em contato direto com o paciente, são especialmente suscetíveis a altas taxas de Burnout, principalmente aqueles que trabaIham em instituições hospitalares, Afinal, a saúde laboral dos profissionais de saúde pode repercutir tanto na qualidade da atenção prestada como no seu grau de formação. 
Por isso propõem-se estudos futuros com o profissional Enfermeiro e que as instituições estejam mais preocupadas com a qualidade de vida, o bem-estar e a saúde física e mental de seus colaboradores devido ao fato de que o Burnout produz sérias repercussões, tanto no âmbito laboral como pessoal, proporcionando a estes suportes, físico e psicológico, para prevenir o desenvolvimento desta patologia.

\section{REFERÊNCIAS}

AVELLAR, L. Z.; IGLESIAS, A.; VALVERDE, P. F. Sofrimento Psíquico em Trabalhadores de Enfermagem de uma Unidade Oncológica. Psicologia em Estudo, Maringá, v.12, 2007.

CAMPOS, R. G. Burnout: uma revisão integrativa na enfermagem oncológica. Ribeirão Preto, 2005. Tese (pós-graduação em enfermagem). Universidade de São Paulo, USP.

CARLOTTO, M. S. O impacto de variáveis sociodemográficas e laborais na Síndrome de Burnout em técnicos de enfermagem. Revista Brasileira de Psicologia Hospitalar, Rio de Janeiro, v. 14, 2011, p. 165-175.

CINTRA, H. S. E.; SOUSA, A. A. L.; LAZZAROTTO, E. M. et al. Fatores que prejudicam o trabalho do enfermeiro que atua em hospital. Seminário Internacional "Experiências de Agendas 21: Os Desafios do Nosso Tempo", Ponta Grossa, 2009.

CODO, W; MENEZES, I. V. 0 que é burnout? . Educação: Carinho e Trabalho. 3 Petrópolis, Rio de Janeiro: Vozes, 2002, p. 237-254.

COSTA, F. M.; VIEIRA, M. A.; SENA, R. R. Absenteísmo relacionado a doenças entre membros da equipe de enfermagem de um hospital escola. Revista Brasileira de Enfermagem, v. 62, n.1, 2009, p. 38-44.

CUMBE, V. F. J. Síndrome de Burnout em Médicos e Enfermeiros Cuidadores de Pacientes com Doenças Neoplásicas em Serviços de Oncologia. Porto, 2010. Dissertação (mestrado em Psiquiatria e Saúde Mental). Faculdade de Medicina Universidade do Porto, FMUP.
FERREIRA, N. M. L. A. A difícil convivência com o câncer: Um estudo das emoções na enfermagem oncológica. Revista Escola de Enfermagem da USP, São Paulo, v. 30, n. 2, 1996, p. 229-253.

JANK, M. A.; CAUDURO, A. B.; NASCIMENTO, A. A. et al. Enfermagem: Um olhar a cerca da Síndrome de Burnout. Centro Universitário Franciscano, 2012.

JODAS, D. A.; HADDAD, M. C. L. Síndrome de Burnout em trabalhadores de enfermagem de um pronto socorro de hospital universitário. Revista Acta Paulista de Enfermagem, São Paulo, v. 22, n. 2, 2009, p. 192-197.

MATUBARO, R. C. A.; LUNARDELLI, M.C.F.; ELLARO, A. M. et al. A Síndrome de Burnout em profissionais da saúde: Uma revisão bibliográfica. Faculdade de ciências-psicologia, Bauru, 2010, p. 981-984.

MICHAELIS. Mini-dicionário inglês-portugûes e portugûes-inglês. São Paulo: Melhoramentos, 1989.

MINISTÉRIO DA SAÚDE. Doenças Relacionadas ao Trabalho: Manual de Procedimentos para os serviços de saúde. Brasilía, 2001

MUROFUSE, N. T.; ABRANCHES, S. S.; NAPOLEÃO, A. A. Reflexões sobre estresse e Burnout e a relação com a enfermagem. Revista Latino Americana de Enfermagem, São Paulo, v. 13, n. 2, 2005, p. 255-261. 
NOGUEIRA, M. L. F. Afastamentos por Adoecimento de Trabalhadores de Enfermagem em Oncologia. Rio de Janeiro, 2007. Dissertação (pós-graduação em enfermagem). Universidade Federal do Estado do Rio de Janeiro, UNIRIO.

O GLOBO ON LINE. Doenças ocupacionais - Síndrome de Burnout afeta grande número de profissionais. Revista Proteção, Hamburgo, 2010. Disponível em: <http://www.protecao.com.br/noticias/doencas_ ocupacionais/sindromede/burnout/afeta/grande/numero/de/profissionais/JyjaJaji>. Acesso em: 25 abr. 2013.

PIRES, D. A.; BRANDÃO, M. R. F.; MACHADO, A. A. Síndrome de Burnout no esporte. Universidade São Judas Tadeu, USJT, Rio Claro, v. 11, n. 3, set./dez, 2005., p. 147-153.

RAMALHO, M. A. N.; MARTINS, M. C. F. N. Vivências de profissionais de saúde da área de oncologia pediátrica. Psicologia em Estudo, Maringá, v. 12, n.1, jan./ abril, 2007, p. 123-132.
RODRIGUES, W. C. Metodologia Científica. Pacambira, 2007.

RODRIGUES, A. B.; CHAVES, E. C. Fatores estressantes e estratégias de coping dos enfermeiros atuantes em oncologia. Revista Latino Americana de Enfermagem, São Paulo, v. 16, n. 1, jan./fev., 2008.

SILVA, J. L. L.; DIAS, A. C.; TEIXEIRA, L.R. Discussão sobre as causas da Síndrome de Burnout e suas implicações à saúde do profissional de enfermagem. Portal das Revistas Científicas em Ciências da Saúde (AQUICHAN), Colombia, v. 12, n.2, agost., 2012, p. 144-149.

SOUZA, W. C.; SILVA, A. M. M. A influência de fatores de personalidade e de organização do trabalho no burnout em profissionais de saúde. Revista Estudos de Psicologia, Campinas, v.19, n.1, 2002, p.37-48.

VIANA, T. S. O estresse da enfermagem no setor oncológico. Revista Científica Onlinez, Maringá, 2010.
1 Possui graduação em Enfermagem pela Universidade Tiradentes (2011). Está cursando Pós Graduação em Enfermagem Oncológica pela Universidade Tiradentes. Atua como Enfermeira no Centro de Atenção PsicossocialCAPS I- No município de Neopólis-Se. Silva.mayara04@hotmail.com

2 Bacharel em Enfermagem com título de Licenciatura em Enfermagem pela Universidade Federal Fluminense (UFF);Especialista em Enfermagem Cardiológica pela Universidade Federal do Rio de Janeiro (UFRJ); Mestranda em Saúde e Ambiente pela Universidade Tiradentes. Colaboradora da Sociedade de Terapia Intensiva de Sergipe (SOTISE). Responsável pelo projeto de extensão Xô Piolho. Fernanda.unit@yahoo.com.br 